\title{
LA INNOVACIÓN Y LA SOSTENIBILIDAD EN LA UNIVERSIDAD-EMPRESA
}

\author{
INNOVATION AND SUSTAINABILITY AT THE UNIVERSITY - ENTERPRISE
}

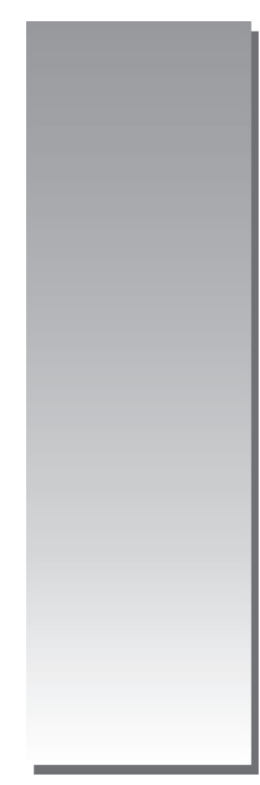

Teresa J. Ríos Delgado* triosd@unmsm.edu.pe

[RECEPCIÓN: JULIO DEL 2014/CONFORMIDAD: SETIEMBRE DEL 2014]

\section{RESUMEN}

La presente investigación correlaciona el tema de la innovación y la sostenibilidad en la universidadempresa, con el objetivo de determinar la relación entre ambas, puesto que se hace imprescindible establecer una gestión que diseñe e implemente una transformación de procesos (educativos) y productos (fines de la universidad) mediante la innovación, como una estrategia empresarial.

El tipo de investigación es llamada aplicada porque pretende enfrentar el problema proponiendo alternativas de solución e igualmente es una investigación documental.

Como principal conclusión se determinó que los nuevos procesos de aprendizaje compartidos por diversas comunidades de estudiantes distantes en el espacio y las empresas deben estar fundados en propuestas, para que estos estudiantes puedan realizar trabajos de investigación en las empresas, utilizando las nuevas tecnologías.

Palabras claves: Innovación, sostenibilidad, universidad, empresa.

\section{ABSTRACT}

This research has been developed in order to obtain a correlation between innovation and sustainability at the university-enterprise, having as an objective to define a relationship between both constructs, because it is essential to settle up a management, which must design and carry through (educational) process and products (university's goals) by means of innovation as a management strategy.

This study is descriptive, explicative and a documental research. As main conclusion it was settled that new processes of shared learnings by several students' communities apart by distance and the enterprises' must be founded in proposals in order to develop research between students and enterprises using new technology.

Keywords: Innovation, sustainability, university, enterprise.

\footnotetext{
* Doctora en Educación, Doctora en Gestión y Desarrollo, Magíster en Educación -Docencia en el Nivel Superior-. Traductora e Intérprete. Bachiller en Derecho. Profesora Auxiliar y Docente Investigadora. Docente de Pre y Posgrado en investigación.
} 


\section{INTRODUCCIÓN}

El presente trabajo analiza el papel fundamental que juega la innovación y la sostenibilidad en la universidad-empresa, en un contexto, en el cual la educación superior en nuestra sociedad se encuentra afectada por, sobre todo, cambios tecnológicos impuestos por la globalización, convirtiendo la demanda de educación superior en un nicho rentable de negocios. Se debe establecer a esta nueva "universidad" en un agente socializador, que crea valores, patrones morales y éticos, que construye y desarrolla procesos sociales y culturales.

Luego de una revisión y precisión de conceptos sobre la innovación con base en la introducción en el mercado (educativo) de un nuevo bien o servicio y la sensibilización de la comunidad universitaria, se argumenta cuál es la relación de la innovación y la sostenibilidad en la universidad-empresa, cumpliéndose el objetivo de establecer dicha relación y aceptándose igualmente la hipótesis; con la entrevista a profundidad como su base metodológica en la recolección de datos.

Es así que seguimos en el planteamiento de una sostenibilidad que complemente a la universidad como institución empresarial e institución educativa para controlar y gestionar el avance científico-técnico que determina la relatividad de las ciencias establecidas por la comunidad científica, lográndose una universidad-empresa responsable frente a las funciones educativas inherentes.

\section{PLANTEAMIENTO DEL PROBLEMA}

En los últimos diez años, gracias a la apertura al libre mercado, el desarrollo económico y social de nuestro país está cambiando a base de factores exógenos propios de los intereses de la "globalización". Es así que nos situamos en un contexto en el cual el conocimiento es la materia prima de los procesos cognitivos en la educación -especialmente en la educación superior universitaria, convirtiéndola en una universidad-empresa. Por su naturaleza, la universidad debería ser el motor del progreso y bienestar de nuestra sociedad, que crea valores, patrones morales y éticos, que construye y desarrolla procesos sociales y culturales pero en esta crisis de valores como la actual, se acrecienta más aún sus funciones de formación y de investigación, como piedra angular en la resolución de los problemas que estamos enfrentando.
Situándonos entonces en la universidad, es imprescindible establecer una gestión que diseñe e implemente una transformación de procesos (educativos) y productos (fines de la universidad) mediante la innovación, como una estrategia empresarial. Hemos comprobado que se prioriza a la empresa antes que a la educación, fenómeno que está avanzado al interior del país, trayendo consigo un atraso no solo en la educación sino también en la sostenibilidad social, económica, política, etc., de nuestra sociedad. Es así que denotamos: ¿Cuál es la relación de la innovación y la sostenibilidad en la universidad-empresa?, fundamentando nuestra hipótesis en que la demanda de educación superior es un nicho rentable de negocios, por lo cual se debe implementar un modelo de excelencia en función de una visión de largo plazo, destinada a cambiar el status de la universidad-empresa.

Sin embargo, una de las limitaciones encontradas es el proceso de acreditación -necesidad indiscutible- en el que se encuentran algunas universidades que disimula las falencias encontradas en la medición o simplemente en el desarrollo de estas.

Se analizó la variable innovación, en función de la introducción en el mercado (educativo) de un nuevo bien o servicio y sensibilización de la comunidad universitaria, y la variable sostenibilidad, según a las políticas de sostenibilidad, responsabilidad social y docencia e investigación.

\section{OBJETIVOS}

En la presente investigación se estableció la relación de la innovación y sostenibilidad en la Universidad-Empresa, mediante la entrevista por juicio de expertos; sin embargo, el diseño de un modelo de gestión de procesos, gestión de la actitud de las personas y la gestión integral de la calidad, implicaría un trabajo a profundidad, pero no se tuvo los recursos económicos para llevarlo a cabo.

\section{JUSTIFICACIÓN}

La principal ventaja competitiva de las organizaciones exitosas reside en la calidad de su gestión, por lo tanto la innovación organizacional, tecnológica y comercial justifica el presente estudio, logrando un posicionamiento en el mercado, aumentando su eficacia competitiva y el valor que perciben los clientes -estudiantes- lográndose el efecto boomerang a los demás estamentos 
-autoridades, docentes, administrativos- y por ende la sociedad; este fenómeno se plasma en la sostenibilidad de la universidad-empresa.

\section{HIPÓTESIS}

La relación de la innovación y la sostenibilidad es directa en la universidad-empresa, lo que conlleva que una mayor innovación, mayor será la sostenibilidad.

\section{METAS ESPECÍFICAS}

Que se determinen ventajas competitivas según la promoción de una cultura y valores organizacionales coherentes con el modelo de la sostenibilidad, tales como apertura, participación, trabajo en equipos, colaboración, responsabilidad y procesos democráticos, solidaridad, compromiso, justicia, contribución, consenso, persistencia, equidad, sensibilidad, y honestidad.

Que se asuma una responsabilidad social empresarial en la cual la universidad-empresa se oriente no solo hacia la maximización de los beneficios educativos, sino también al bienestar social y la investigación científica.

\section{MARCO TEÓRICO DEL TRABAJO}

\section{La innovación}

Es todo cambio que está basado en conocimiento y que genera valor. Esto expresa que la innovación tiene al valor como su meta, al cambio como su vía y al conocimiento como su base.

Así pues, la innovación se orienta a aumentar el valor tanto de la propia empresa como de su oferta. En el primer caso, deberá contribuir a una mejor cuenta de resultados, una mejor posición bursátil o una mejor imagen y, en el segundo, a un mejor comportamiento en el mercado de su oferta, reflejado en el incremento de su cuota o en una mejor aceptación de precios unitarios más elevados.

El cambio es la vía para la innovación. En la empresa este cambio debe tener lugar en sus bienes o servicios, los procesos de producción o provisión, su forma de organizarse o la manera de relacionarse con sus clientes o suministradores (COTEC, 2010). Porter (Porter, 1990), por ejemplo, es categórico en reconocer el importante papel de la innovación cuando menciona que "la competitividad de una nación depende de la capacidad para innovar y mejorar".

\section{La cultura innovadora}

La cultura innovadora de una empresa supone la existencia de un conjunto de valores, hábitos y conocimientos que facilitan el desarrollo y éxito de actividades innovadoras, impulsados desde la más alta dirección y sobre todo compartidos por todos los trabajadores.

Se asienta en un estilo de dirección participativo y capaz de promover la confianza, la colaboración, el aprendizaje mutuo y la mejora personal. La dirección participativa facilita la cooperación de los colaboradores en la fijación de objetivos y en la toma de decisiones, haciéndoles de este modo cómplices del compromiso y aumentando con ello su motivación para contribuir al logro de los resultados previstos. Los proyectos innovadores incluyen muchas actividades que se realizan en equipo, en colaboración con personas de muy diferentes perfiles y procedencias, y pueden resultar muy fortalecidos si entre ellas se establece un intercambio de experiencias basado en la confianza mutua. La innovación requiere además habilidades y conocimientos en muy distintos aspectos, que deben verse incluidos en los planes de formación de la empresa innovadora y que los trabajadores más emprendedores deben buscar adquirir.

La cultura innovadora requiere también una asignación flexible de responsabilidades, de forma que los trabajadores puedan intervenir en las iniciativas innovadoras más propicias a sus capacidades.

Luego, apreciamos que el cambio en la cultura es muy importante para la innovación, y situándolo en el campo educativo Carbonell (Canal de Leon, 2002), quien entiende la innovación educativa como:

"(un) conjunto de ideas, procesos y estrategias, más o menos sistematizados, mediante los cuales se trata de introducir y provocar cambios en las prácticas educativas vigentes. La innovación no es una actividad puntual sino un proceso, un largo viaje o trayecto que se detiene a contemplar la vida en las aulas, la organización de los centros, la dinámica de la comunidad educativa y la cultura profesional del profesorado. Su propósito es alterar la realidad vigente, modificando concepciones y actitudes, alterando métodos e intervenciones y mejorando o transformando, según los casos, los procesos de enseñanza y aprendizaje. La innovación, por tanto, va asociada al cambio 
y tiene un componente - explícito u ocultoideológico, cognitivo, ético y afectivo. Porque la innovación apela a la subjetividad del sujeto y al desarrollo de su individualidad, así como a las relaciones teoría-práctica inherentes al acto educativo".

La mayoría de la literatura en innovación en la educación desarrolla el nivel básico regular (escolar) -base en la cual también fundamentamos el desarrollo de la presente investigación. Por ejemplo (Ríos, 2008) :

\section{- Modelo de Investigación y Desarrollo}

Este modelo, totalmente orientado al proceso educativo, desarrolla las primeras innovaciones educativas en las ciencias, tecnología e industria de los países desarrollados. Su enfoque lógico y racional busca resolver un determinado problema, detectado por profesionales externos -individuales e institucionales- a la escuela, a través de una estrategia que se considera como la más adecuada.

Este modelo encaja en los objetivos de la universidad peruana de hoy, donde se hace imprescindible el trabajo biunívoco entre docente y estudiante ya que es asimétrico, dado el grado de colaboración entre los que realizan el diseño de la innovación y los ejecutantes.

Así, los especialistas (diseñadores) deciden qué es lo conveniente de poner en marcha y los docentes son considerados técnicos que ejecutan las diversas acciones contempladas en la mejora elaborada por aquellos. Estas acciones plantean una secuencia lineal y cada actividad presenta un fino detalle para su ejecución, por lo que la función del profesor se limita al cumplimiento racional de lo previamente establecido por otros, independiente de su interés y necesidad de cambiar. Las innovaciones exitosas desarrolladas a la luz de este modelo se validan externamente a través de su difusión y adopción por otros interesados. Estas fases incorporan actividades de diseminación, demostración, prueba, implantación e institucionalización definitiva (Morrish, 1978).

\section{- Modelo de Interacción Social}

Se basa en la observación y juicio de los posibles usuarios de la innovación, que es formulada y ejecutada en una realidad escolar diferente a la de estos. Los posibles adaptadores, a través de la relación personal y profesional que establecen con otros innovadores, toman conocimientos de la ejecución de una determinada innovación, estimando la posibilidad de replicarla en su propio ámbito educativo, al poseer una problemática similar al contexto donde se aplica esta. Este modelo es utilizado en las escuelas demostrativas y presenta las siguientes fases: toma de conciencia, interés evaluación, ensayo y adopción.

\section{- Modelo Organizativo}

El modelo organizativo se plantea desde un enfoque cultural, considerando a la escuela como una organización que posee una cultura propia y distintiva. Está basado en la dinámica de las organizaciones, cuya característica general se relaciona con la constitución de entramados de personas y grupos que se vinculan de diversas formas al interior del centro educativo, lo que puede favorecer o no el cumplimiento de los fines y objetivos institucionales (Zaltman, et al., 1977 y Brow y Moberg, 1980, en Fernández y Puente, 1992). Sus principales fases de este modelo son: planificación, adopción, ejecución y evaluación.

\section{- Modelo Solución de Problemas}

Este modelo también se presenta desde una perspectiva cultural y tiene como punto de partida y como eje central a los profesores, en tanto que son los que definen las necesidades iniciales y actúan solos o con asesores. Estos pretenden reestructurar internamente las condiciones desfavorables que presenta la labor educativa de la escuela y su participación es esencial para el logro de la innovación formulada.

El agente externo puede asumir el rol de colaborador de la experiencia de mejoramiento, pero no tiene el protagonismo que se espera de los profesores. La interacción y reconocimiento mutuo de estos es la condición necesaria para el éxito de la innovación aplicada (Morrish, 1978).

\section{Sostenibilidad}

En el año 1987 (Duran, 2013), la publicación del libro "Nuestro Futuro Común”, más conocido como informe Brundtland, popularizó el concepto de desarrollo sostenible, que bajo otras acepciones como, por ejemplo, ecodesarrollo, se venía utilizando desde los años setenta. Con este concepto, se plantea la búsqueda de progreso 
económico que no descuide aspectos cualitativos, tales como la calidad de vida o la preservación del medio ambiente sin olvidar un compromiso ético con las generaciones venideras.

A partir del informe Brundtland, el mundo es concebido como un sistema global cuyas partes están interrelacionadas, considerándose el concepto de desarrollo sostenible como un proceso multidimensional que afecta al sistema económico, ecológico y social, pasando a ser una variable a tener en cuenta en las decisiones de política económica.

Sin embargo, en los últimos años, una de las cuestiones más preocupantes ha sido el conocer si realmente se siguen pautas de sustentabilidad, es decir, si se tienen indicadores que nos alerten sobre la evolución positiva o negativa de este proceso. Aunque la ambiguiedad del propio concepto dificulta esta tarea, se han ido elaborando algunos indicadores que muestran aspectos de las tres dimensiones mencionadas anteriormente.

El concepto de desarrollo sostenible tal y como se definió anteriormente ha sido clasificado de ambiguo, considerándose más “ (...) un deseo general pero sin precisar mucho el contenido ni el modo de llevarlo a cabo" (Naredo, 1996, p. 529). En esta línea, Rivas se pregunta también "cuál es la virtualidad del desarrollo sostenible o, lo que viene a ser lo mismo, para qué se acuña y se defiende un concepto vacío, ambiguo (...), la respuesta es aparentemente sencilla: el desarrollo sostenible no es, propiamente, un concepto, sino un metaconcepto. La virtualidad de la idea, del principio general, radica, en primer lugar, en algo que es evidente: el consenso que provoca en torno a sí. Desde este momento, la protección del medio (...) requiere un acuerdo general, encontrándose íntimamente ligado a la implantación de un nuevo orden económico mundial y a la democratización de las organizaciones internacionales" (Rivas, 1997 p. 46).

No obstante, a pesar de la ambiguiedad del concepto, si es posible aceptar un cierto consenso. Por ejemplo, hay unanimidad respecto a su interpretación en cuanto que se entiende la sustentabilidad como la capacidad para continuar en el futuro realizándose, además, este proceso como la interacción entre tres sistemas: el ecológico (biológico), el económico y el social (Daly, 1995, p. 21).
En abril de 2009, se celebró en la ciudad alemana de Bonn la Conferencia sobre EDS, cuyos participantes suscribieron la "Declaración de Bonn”-http://www.esd-world-conference-2009. org/fileadmin/download/ESD2009_Bonn DeclarationESP.pdf-, la cual denotó: "La educación para el desarrollo sostenible sigue progresando de manera desigual y exige diferentes enfoques en contextos diferentes. Sin duda, en los próximos años, será necesario que tanto los países desarrollados como los países en desarrollo, la sociedad civil y las organizaciones internacionales realicen esfuerzos para... apoyar la incorporación de temas de desarrollo sostenible mediante un enfoque integrado y sistémico, en todos los niveles de la educación formal, no formal e informal, en particular mediante la elaboración de métodos pedagógicos eficaces, la formación de docentes, los planes y programas de estudios, los materiales didácticos y el desarrollo del liderazgo educativo.

Y Unesco ha publicado recientemente el informe titulado "Strategy for the United Nations Decade of Education for Sustainable Development, 2005-2014", en el que enumera desafíos claves en el ámbito de Europa y Norte América: “...la falta de competencia en este tema, en particular en el sector educativo; avanzar hacia la interdisciplinariedad y la naturaleza holística de la EDS; la carencia de un consenso sobre la comprensión común de la EDS; o la confusión sobre la comprensión de las diferencias entre la educación ambiental y la educación para el desarrollo sostenible...".

\section{Responsabilidad social}

La responsabilidad social tiene los siguientes alcances (Lopez, 2007)

- La Responsabilidad Social compete a todo tipo de Organizaciones (empresas, OSC, Estado, Universidad, etc.).

- Las organizaciones deben ser socialmente responsables al momento de tomar sus decisiones y realizar sus actividades.

- Se incorpora el concepto de ciudadanía organizacional responsable.

- La organización socialmente responsable desarrolla su gestión con un comportamiento basado en valores: ética y transparencia son dos palabras clave.

- La responsabilidad social debe formar parte de la estrategia de la organización y por ello la abarca en su totalidad. 
En el Libro Verde, publicado por la Comisión Europea en 2001, se define la responsabilidad social de la empresa como "la integración voluntaria, por parte de las empresas, de las preocupaciones sociales y medioambientales en sus operaciones comerciales y sus relaciones con sus interlocutores". Según esta definición, ser "socialmente responsable" va más allá de cumplir estrictamente la legislación en los países donde la empresa opera. Se trata de invertir más y mejor en aspectos tales como el desarrollo del capital humano, la protección medioambiental o el fomento de las sociedades donde la empresa realiza sus actividades; significa también, como veremos más adelante, aprovechar las oportunidades que esta visión ofrece para mejorar la competitividad e, incluso, identificar nuevas oportunidades de negocio.

Significa que, más allá del beneficio económico, la principal función de una empresa consiste en crear valor con la producción de bienes y servicios que respondan a la demanda de la sociedad y generar de este modo beneficios sostenibles para sus propietarios y accionistas, así como bienestar para la sociedad en general, en particular gracias a un proceso continuo de creación de empleo. De hecho, cada vez son más los empresarios que ven en un comportamiento responsable un camino cierto para alcanzar el éxito comercial y proporcionar beneficios duraderos para sus accionistas. De ahí que algunas de las empresas líderes en todo el mundo hayan apostado firmemente por este camino como garantía de su supervivencia a largo plazo (Alfaya, s/a).

\section{Docencia e Investigación}

Con el inicio del proceso de acreditación, todos los países y universidades han comenzado a poner en práctica evaluaciones institucionales de los logros de la investigación, pasando de ser una responsabilidad individual a un interés colectivo de la organización y los departamentos (Henkel, 1999).

Entre las dimensiones de los estándares de acreditación, se puede observar a la docencia e investigación como correlacional, ya que estamos en el contexto en el cual no puede haber un docente que no investigue.

Braxton (Braxton, 1996) propone tres posibles perspectivas para esta conexión:
- Nula, cuando no existe relación.

- De conflicto, cuando la relación es negativa.

- Complementaria, cuando existe similaridad entre la enseñanza y la investigación.

Sin embargo, Vidal y Quintanilla (2000) proponen enfocar el problema como un sistema dinámico en el que es posible encontrar diferentes bucles entre enseñanza e investigación. En algunas circunstancias, para estos autores, la relación será positiva y se posibilitará la transferencia, mientras en otras será negativa y se creará interferencia.

Clark (1997) realizó un análisis de cómo poder integrar la investigación y la docencia y argumentó que la investigación tiene, por supuesto, un lugar en el ámbito de la enseñanza y que este concepto no es nuevo ni en la teoría ni en la práctica. Sin embargo, para este autor, esta fuerte conexión se encontraría sobre todo en los programas de posgrado. En este marco no hay espacio para una tesis de incompatibilidad. Este análisis no pierde de vista los programas de licenciatura, en los que la actividad docente suele tener lugar con grupos numerosos, en los que abundan las clases expositivas centradas en el profesor.

En este contexto, si se realiza investigación suele verse como una actividad separada de la docencia. En este ámbito, encuentra Clark un cierto nivel de incompatibilidad y sugiere que se le preste una especial atención.

\section{MÉTODO}

Esta investigación sobre la relación entre la innovación y sostenibilidad en la universidad- empresa es una investigación aplicada porque se va a enfrentar el problema proponiendo alternativas de solución.

El diseño básico de esta investigación es no experimental - transeccional - explicativo correlacional. es no experimental, debido a que no se harán pruebas; es decir en esta investigación las variables no van a ser manipuladas. Es transeccional, porque nuestro objeto de estudio va a ser analizado en un periodo determinado de tiempo. Es explicativo correlacional, porque se trata de encontrar evidencias de cómo la variable independiente innovación se relaciona con la sostenibilidad. 


\section{RESULTADOS}

Estamos ad portas de un cambio en la educación superior, que va a quebrar las actividades que distinguen a una organización de otra (universidad) en este mercado globalizado. Por lo tanto, la universidad-empresa debe desarrollar una actitud creativa y renovadora y capacidad de liderazgo para relacionarse directamente con los cambios existentes. La investigación es la actividad básica e inherente en la universidad y esta es sinónimo de ciencia. Mediante la innovación se debe constituir procesos encaminados a la construcción colectiva de temas académicos en los cuales se fundamentará el sistema universitario de investigación. Es así que la sostenibilidad será en el día a día, ya que la educación superior debe ser un nicho rentable de negocios y de inversión empresarial. La universidad-empresa, como agente socializador, crea valores, patrones morales y éticos, que construye y desarrolla procesos sociales y culturales.

\section{DISCUSIÓN}

Al encontrarnos en un contexto en el cual la educación superior en nuestra sociedad se encuentra afectada por los cambios científicos, y sobre todo tecnológicos, convirtiendo la demanda de educación superior en un nicho rentable de negocios, el aporte científico que se espera obtener es el diseño de un modelo de excelencia basado una visión de largo plazo, destinada a cambiar el status quo de la universidad-empresa, como agente socializador que crea valores, patrones morales y éticos, que construye y desarrolla procesos sociales y culturales.

\section{CONCLUSIONES}

- La relación entre la innovación y la sostenibilidad en la universidad-empresa es directa, pues los nuevos procesos de aprendizaje compartidos por diversas comunidades de estudiantes distantes en el espacio y empresas se fundan en propuestas para que los estudiantes puedan realizar trabajos de investigación en las empresas, con las nuevas tecnologías.

- A pesar del reconocimiento a la universidad como factor fundamental en el desarrollo humano y empresarial por parte de los actores económicos, aún no hay un empoderamiento que situé a la universidad-empresa en el contexto de innovación y sostenibilidad.

- La universidad-empresa, por lo tanto, debe crear contextos de enseñanza-aprendizaje en los que el trabajo académico esté íntimamente ligado a la vida y al contexto empresarial en que está inmersa la universidad.

\section{REFERENCIAS BIBLIOGRÁFICAS}

Alfaya, V. (s/a). www.peru2021.org. Obtenido de www.peru2021.org/repositorioaps/0/0/par/ articulorsebiblioteca/alfaya_rse.pdf

Arias, F. (2006) El proyecto de investigación: Introducción a la metodología científica. 5ta ed. Caracas. Episteme

Braxton, J. (1996). Contrasting perspectives on the relationship between teaching and research. Wiley Periodicals.

Burmester, H. (2006). Crecimiento económico como factor importante para la sostenibilidad.

Canal de León, P. (2002). La innovación educativa. Madrid: AKAL.

Clark, A. (1997). Being there. Putting Brain, Baody and world together again. Cambridge: The MIT Press.

Castro, M (2003). El proyecto de investigación y su esquema de elaboración. 2ed. Caracas. Uyapal

Colom, A. (2000). Desarrollo sostenible y educación para el desarrollo. Barcelona: Octaedro.

COTEC. (2010). La innovación en sentido amplio. Madrid: Graficas Arias Montano .

Correa, María Emilia (2004). Responsabilidad social empresarial: una nueva forma de hacer negocios. Revista Futuros No. 6. Volumen 2.

Chiavenato, Idalberto (2006). Introducción a la Teoría General de la Administración. Séptima Edición. McGraw-Hill Interamericana.

Daly, H. y. (1995). Significado, conceptualización y procedimientos operativos del desarrollo sostenible: posibilidades de aplicación a la agricultura. Madrid. 
Durán, G. (12 de Diciembre de 2013). pendientedemigracion.ucm.es. Obtenido de pendientedemigracion.ucm.es/info/ec/jec7/pdf/ com1.6.pdf

Drucker, P.F. (1986). La innovación y el empresariado innovador. Buenos Aires, Editorial Sudamericana, S.A.

Gómez Vieites, Álvaro (2004). Sistemas de información: herramientas prácticas para la gestión empresarial. México, Alfaomega.

Henkel, M. (1999). The modernisation of research evaluation: The case of UK. Netherlands: Kluwer Academic Publishers.

Hernández, Fernández y Baptista (2010). Metodología de la Investigación. México, Mc. Graw Hill.

Kuang-tsae, H., Lee, Yang, W. \& Wang, R. (2000). Calidad de la información y gestión del conocimiento. Madrid. Editorial AENOR.

López, M. (Junio de 2007). www.ccee.edu.uy. Obtenido de www.ccee.edu.uy/investigacion/ lineas/etica/ATT00322.PDF

Mejía, Elías (2005). Metodología de la investigación científica. Lima, Centro de Producción Editorial de la UNMSM.
Morrish, I. (1978). Cambio e innovacion en la enseñanza. Salamanca: ANAYA.

Pérez Lindo, Augusto y otros (2005). Gestión del conocimiento. Grupo Editorial Norma. Buenos Aires.

Porter, M. (1990). The competitive advantage of the nations. New york: Free Press.

Ríos, D. y. (2008). Proyectos de innovacion educativa. Chile.

Ríos, Teresa y Torres, Charles (2013). La tesis teoría - práctica. Lima, Centro de Producción Editorial de la UNMSM.

Rivas, F. (1997). El proceso de enseñanza/aprendizaje en la situacion educativa. Barcelona: Ariel.

Senge, Peter (2004). La quinta disciplina. Buenos Aires. Ediciones Granica.

Vidal, J. y Quintanilla, M. (2000). The teaching and research realtionship within institutional evaluation. Higher Education. 